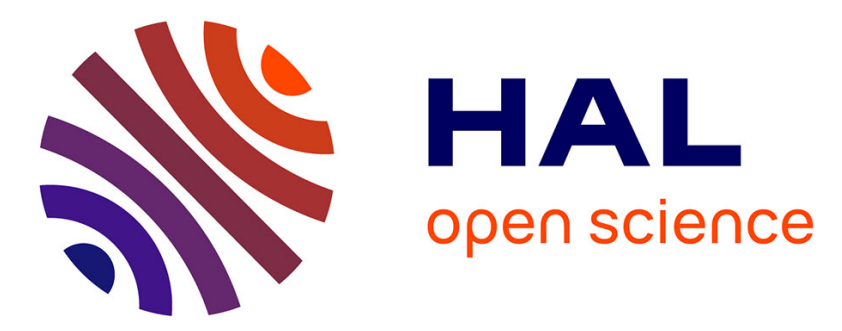

\title{
L'habitat de plaine Néolithique final du Bousquetas à Paulhan (Hérault)
}

Laurent Carozza, Pascal Vialet, Anne Bouchette, Pascale Chevillot

\section{To cite this version:}

Laurent Carozza, Pascal Vialet, Anne Bouchette, Pascale Chevillot. L'habitat de plaine Néolithique final du Bousquetas à Paulhan (Hérault). Bulletin de la Société préhistorique française, 1999, 96 (1), pp.39 - 52. 10.3406/bspf.1999.10907 . hal-02990672

\section{HAL Id: hal-02990672 \\ https://hal.science/hal-02990672}

Submitted on 5 Nov 2020

HAL is a multi-disciplinary open access archive for the deposit and dissemination of scientific research documents, whether they are published or not. The documents may come from teaching and research institutions in France or abroad, or from public or private research centers.
L'archive ouverte pluridisciplinaire HAL, est destinée au dépôt et à la diffusion de documents scientifiques de niveau recherche, publiés ou non, émanant des établissements d'enseignement et de recherche français ou étrangers, des laboratoires publics ou privés. 


\title{
L'habitat de plaine Néolithique final du Bousquetas à Paulhan
} (Hérault)

\author{
Laurent Carozza, Pascal Vialet, Anne Bouchette, Pascale Chevillot
}

\section{Citer ce document / Cite this document :}

Carozza Laurent, Vialet Pascal, Bouchette Anne, Chevillot Pascale. L'habitat de plaine Néolithique final du Bousquetas à Paulhan (Hérault). In: Bulletin de la Société préhistorique française, tome 96, n¹, 1999. pp. 39-52;

doi : https://doi.org/10.3406/bspf.1999.10907

https://www.persee.fr/doc/bspf_0249-7638_1999_num_96_1_10907

Fichier pdf généré le 13/04/2018 


\title{
Résumé
}

Résumé Le site Néolithique final du Bousquetas a été découvert dans la vallée de l'Hérault, sur le tracé du Gazoduc. La fouille a permis de mettre au jour 9 structures en creux dont certaines se rapportent à des silos arasés. L'analyse des macro-restes végétaux recueillis dans les structures FS.2, FS.3 et FS.4 contribue à définir le statut agricole du site. Si les mobiliers recueillis s'avèrent indigents, une datation $\mathrm{Cl} 4$ confirme l'attribution au Néolithique final de cet habitat de plaine. Cette fouille contribue à une meilleure connaissance de l'habitat de la fin du Néolithique. Le site du Bousquetas illustre la complémentarité entre les habitats de hauteur fortifiés, tel celui de Roquemengarde, et de petites unités de plaine à vocation peut-être spécialisée.

\begin{abstract}
Abstract The Late Neolithic site of Bousquetas was discovered in the Hérault valley, along the route of a gas pipeline. The excavations carried out in that area have enabled nine hollow structures to be uncovered, some of which correspond to levelled silos. The analysis of vegetable macro-remains gathered in the FS.2, FS.3 and FS.4 structures contributes to the definition of the agricultural nature of the site. Despite the scarcity of the material collected it has been possible to establish, thanks to a C 14 date, that this dwelling site on the plain was occupied during the Late Neolithic. These excavations have contributed to improving knowledge regarding Late Neolithic settlements. The Bousquetas site shows the complementarity between fortified hilltop sites such as the one found at Roquemengarde and small units on the plains which may have had some specific purpose.
\end{abstract}




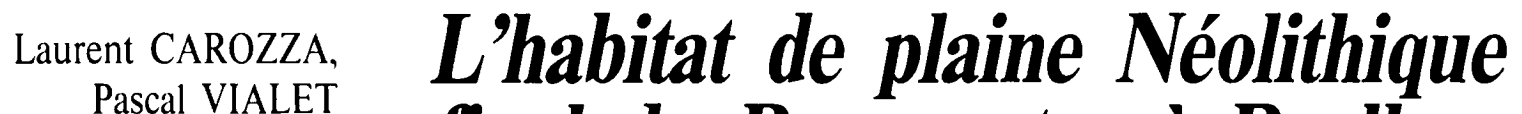 avec la collaboration final du Bousquetas à Paulhan de Anne BOUCHETTE et Pascale CHEVILLOT (Hérault)
}

\begin{abstract}
Résumé
Le site Néolithique final du Bousquetas a été découvert dans la v'allée de l'Herault, sur le trace du Gazoduc. La fouille a permis de mettre au jour 9 structures en creux dont certaines se rapportent à des silos arasés. Lanalyse des macro-restes végétaux recueillis dans les structures FS.2, FS.3 et FS.4 contribue à définir le statut agricole du site. Si les mobiliers recueillis s'avèrent indigents. une datation Cl4 confirme l'attribution au Néolithique final de cet habitat de plaine. Cette fouille contribue à une meilleure connaissance de l'habitat de la fin du Néolithique. Le site du Bousquetas illustre la complémentarite e'mere les habitats de hauteur fortifie's, tel celui de Roquemengarde, ('t de petite's unite's de' plaine à vocation peut-être spécialisée.
\end{abstract}

\begin{abstract}
The Late Neolithic site of Bousquetas was discovered in the Herault vallet, along the route of a gas pipeline. The excavations carricd out in that area have enabled nine hollow structures to be uncovered. some of which correspond to levelled silos. The analysis of vegetable macro-remains gathered in the FS.2. IS.3 and FS.4 structures comtributes to the definition of the agricultural nature of the site. Despite the scarcity of the material collected it has been possible to establish, thanks to a Cl4 date. that this dwelling site on the plain was occupied during the Late Neolithic. These excatations have contributed to improving knowledge regarding Late Neolithic semlements. The Bousquetas site shows the complementarity between fortified hilltop sites such as the one found at Roquemengarde and small units on the plains which mal have had some specific purpose.
\end{abstract}

\section{LE SITE}

\section{Circonstances de la découverte et conditions d'étude}

Le gisement de plein air du Bousquetas se trouve sur le territoire de la commune de Paulhan, à environ $1 \mathrm{~km}$ au nord est de l'agglomération. Il a été découvert à l'occasion de sondages diagnostics engagés sur les formations alluviales de la rive droite de la vallée de l'Hérault traversées par le fuseau d’emprise du gazoduc (fig. 1). Dans la zone accessible, neuf fosses ont été dégagées et fouillées. Ce groupe de structures appartient à une occupation probablement beaucoup plus vaste, partiellement recoupée par l'emprise des travaux. De fait, l’étendue du site n’est pas connue et demeure très diflicile à spécifier.

Le gisement a été signalé par une fosse (FS.1), dégagée par la tranchée de diagnostic. Cette phase initiale a été suivie par un décapage sur environ $70 \%$ de la largeur de l'emprise qui à cet endroit présente un élargissement sensible $(30 \mathrm{~m})$. Cette extension correspond au terrassement imposé pour le franchissement du seuil entre les deux niveaux de terrasse. Ce décapage a été complété par un nettoyage manuel et un marquage des contours des structures. Puis, une levée du plan général a été réalisée. Dans un premier temps il a été préconisé une fouille de $50 \%$ de chaque structure afin de tester leur puissance, leurs caractéristiques stratigraphiques et déterminer les prélèvements à entreprendre. Après relevés des profils et des remplissages. 
la deuxième moitié du comblement a été fouillée dans le but de collecter le mobilier archéologique restant et effectuer les prélèvements anthracologiques et carpologiques indiqués par les tests réalisés sur la première moitié du remplissage'.

\section{Localisation}

Le site est situé dans la plaine alluviale de l'Hérault, sur le rebord d'un niveau supérieur de la moyenne terrasse (Fya) d'altitude 30-40 m. Il domine vers l'est un niveau
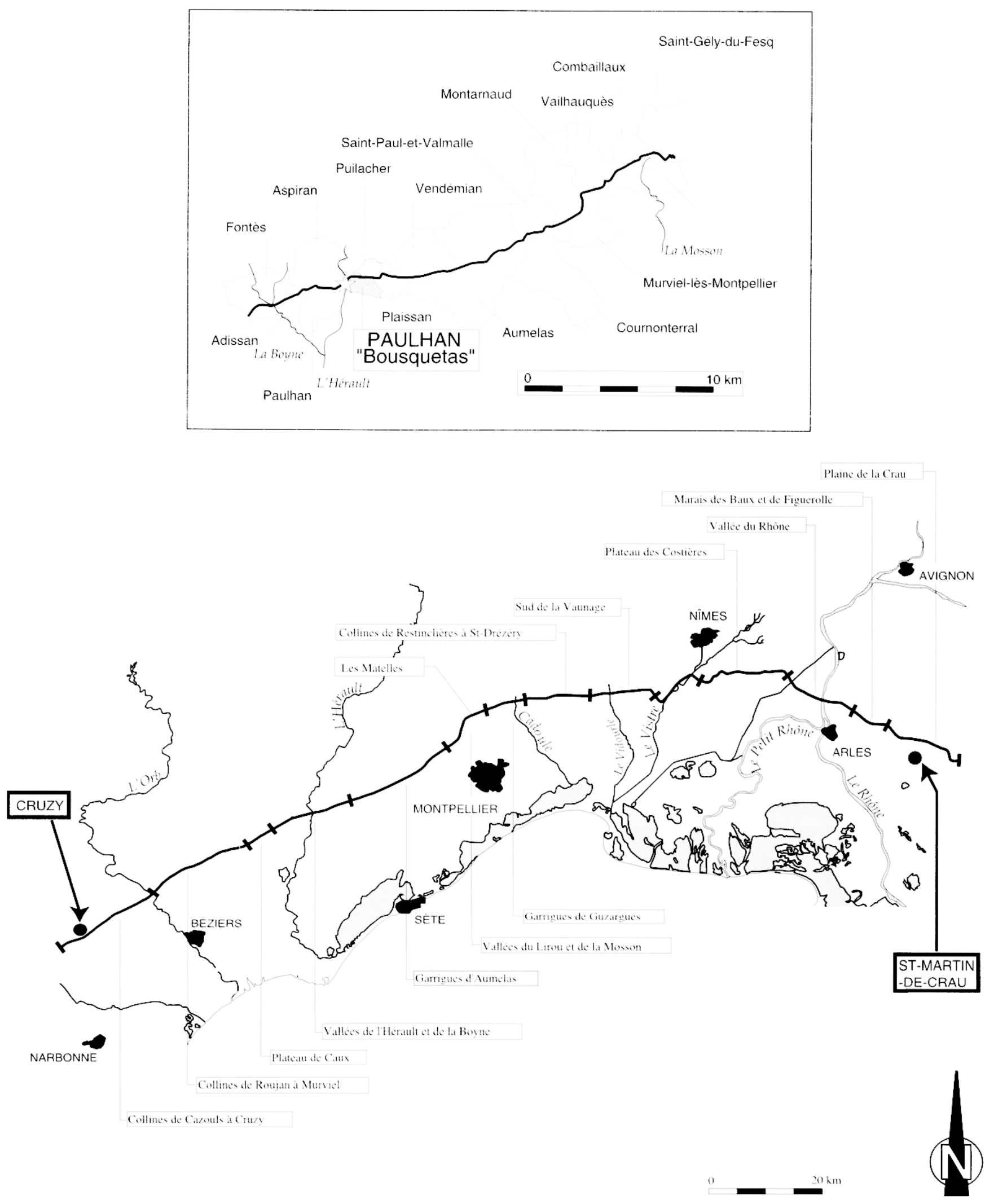

Fig. 1 - Situation du site du Bousquetas a Paulhan. Tracé du Gazoduc " artere du Midi" (dessin L. Loiselier). 
inférieur de la terrasse Fy d'altitude 8-20 m et la basse terrasse de l'Hérault qui coule à environ $700 \mathrm{~m}$. Vers l'ouest, à quelques mètres du gisement, se développe le plateau de Paulhan dont l'altitude moyenne est de $100 \mathrm{~m}$. Le gisement préhistorique occupe un terrain plat. À cet endroit, le substrat miocène est recouvert par 2 à $3 \mathrm{~m}$ de graviers plus ou moins grossiers. La terre végétale, riche en sable et graviers, présente une épaisseur moyenne de $0,40 \mathrm{~m}$. Les sols d'occupations ont été détruits par les labours ainsi que la partie supérieure des structures en creux. Ces différents aménagements ne possèdent aucune relation stratigraphique directe ou indirecte.

\section{DESCRIPTION DES STRUCTURES ET DE LEUR REMPLISSAGE}

\section{La fosse FS.1}

Cette structure en creux se présentait sous la forme d'un indice pédologique de forme grossièrement circulaire (diamètre compris entre $1 \mathrm{~m}$ et $0,90 \mathrm{~m}$ ). La section réalisée a permis de mettre en évidence un creusement conservé sur une puissance de $1 \mathrm{~m}$ (fig. 2). La stratification a été établie du bas vers le haut à l'aide des critères sédimentologiques (couleur et texture du sédiment). $\dot{A}$ la base du remplissage, outre un remaniement noté US.1.6, on remarque la présence d'un sédiment limoneux de teinte brune, comportant de très nombreux graviers (US.1.5). Cette phase du remplissage, qui pourrait pro-parte correspondre à un stade d'érosion des parois, présente une forme légèrement incurvée. L'unité stratigraphique US.1.4 est constituée d'un sédiment argileux, mêlé à des poches d'argile verte. En outre, cette couche comporte de très nombreux apports anthropiques, pouvant suggérer une phase rapide de colmatage de type dépotoir. À l'inverse, l'unité stratigraphique US.1.3 ne comporte quasiment plus d'apports anthropiques. Le sédiment est un limon-sablonneux graveleux comportant également de petits galets. L'accumulation sédimentaire observée le long des parois pourrait résulter de l'effondrement d'une partie du sommet de la structure. L'épisode US.1.2 du remplissage est le plus puissant comprenant des graviers et petits galets. De texture argilo-limoneuse de teinte brun rouge, il comporte à son sommet des galets de bonne dimension. Le sommet du remplissage de texture limono-argileuse de teinte brune comportait également des plages de sable rouge. De toute évidence, les deux dernières unités stratigraphiques correspondent à une phase d'altération du creusement, comme semble l'indiquer la présence de galets et graviers.

La forme générale du creusement ne nous renseigne guère sur sa fonction primaire. Le fond du creusement présente une légère concavité, tandis que les parois sont généralement verticales. On remarque cependant la présence de deux légères dépressions opposées dans la partie supérieure du creusement. Ce dernier pourrait correspondre à une phase d'altération de la structure. De toute évidence la base du remplissage s’apparente à une phase dépotoir à laquelle succède une phase détritique. Cette conformation permet de privilégier l'hypothèse d'un silo effondré. On remarquera également la présence d'argile découverte dans le fond de la fosse pouvant correspondre aux vestiges du bouchon.

\section{La fosse FS.2}

Cette fosse est apparue au décapage sous la forme d'un indice de forme oblongue, d'une longueur de $2.12 \mathrm{~m}$ pour une largeur de $0,90 \mathrm{~m}$. Son remplissage très homogène. conservé sur une profondeur de $0,75 \mathrm{~cm}$, a été scindé en deux unités (fig. 2). L'unité de base (US.2.2) se compose d'un sédiment sablo-limoneux riche en graviers. Le mobilier y est épars. La partie supérieure du comblement présente des caractères sédimentaires analogues. Toutefois, la densité en galets et graviers décroît sensiblement alors que celle des apports anthropiques est en augmentation.

La fonction primaire de cette fosse est difficile à déterminer. Par ses proportions, cette structure s'écarte des canons admis pour les structures de stockage classiques. L’absence de traces de combustion écarte l'hypothèse d'unc fosse à combustion. Sa forme n'évoque pas non plus l'aspect d'une structure d'extraction en général alvéolée, d’autant plus que le substratum ne se prête pas à cette fonction. Nous pouvons, à titre d'hypothèse, suggérer que cette fosse profonde, ouverte, correspond à une forme particulière de stockage en atmosphère libre, sorte de "cave" protégée par une couverture en matériau périssable. Néanmoins, aucune trace ne permet de l'attester. En tout état de cause, cette structure comporte un remplissage secondaire de type dépotoir.

\section{La fosse FS.3}

Cette structure en creux, de plan grossièrement circulaire (diamètre moyen de $0.70 \mathrm{~m}$ ), présente un remplissage conservé sur une profondeur d'environ $0.15 \mathrm{~m}$ (fig. 2). Ce dernier se composait de cendres mêlées à des plages de sédiment sablo-graveleux et occupait de manière homogène l'ensemble de la structure. L'absence de traces de rubéfaction tant sur les parois du creusement que labsence de transformation des matériaux présents dans la fosse permet d'écarter l'hypothèse d'une structure de combustion. La présence en revanche, hormis celle de cendres de très nombreux restes carbonisés suggère un dépôt secondaire de type vidange. Par ses proportions, cette structure s’apparente à une cuvette, structures souvent associées aux foyers domestiques (Gascó, 1981).

\section{La fosse FS.4}

La structure FS.4 présente des caractéristiques analogues à la précédente. D’un point de vue morphologique le creusement présente un plan circulaire d'un diamètre de $0,50 \mathrm{~m}$. Le creusement est conservé sur une puissance de $0,15 \mathrm{~m}$ (fig. 2). Le remplissage correspond à un limon sablono-graveleux mêlé à des plages de limon brun. L'ensemble comporte de nombreux 
restes carbonisés. Cet aménagement se rapporte à une cuvette comblée par des vidanges de foyers.

\section{La fosse FS.5}

Écrêtée par les labours, cette fosse présente un plan circulaire d'un diamètre d'un mètre. Son creusement est conservé sur une hauteur de $0,50 \mathrm{~m}$ (fig. 3). Les parois nettement concaves créent dans la partic supérieure de la structure l'amorce d'une voûte. Ce profil en "cloche" peut être jugé caractéristique des silos. Le comblement conservé est constitué d’un limon brungris englobant des graviers et des blocs (US.5.1). La richesse du mobilier céramique contenu dans ce comblement traduit la dévolution secondaire de cette excavation, en tant que dépotoir.

\section{La fosse FS.6}

Il s`agit d’une structure en creux totalement détruite par les travaux agricoles. La faible épaisseur

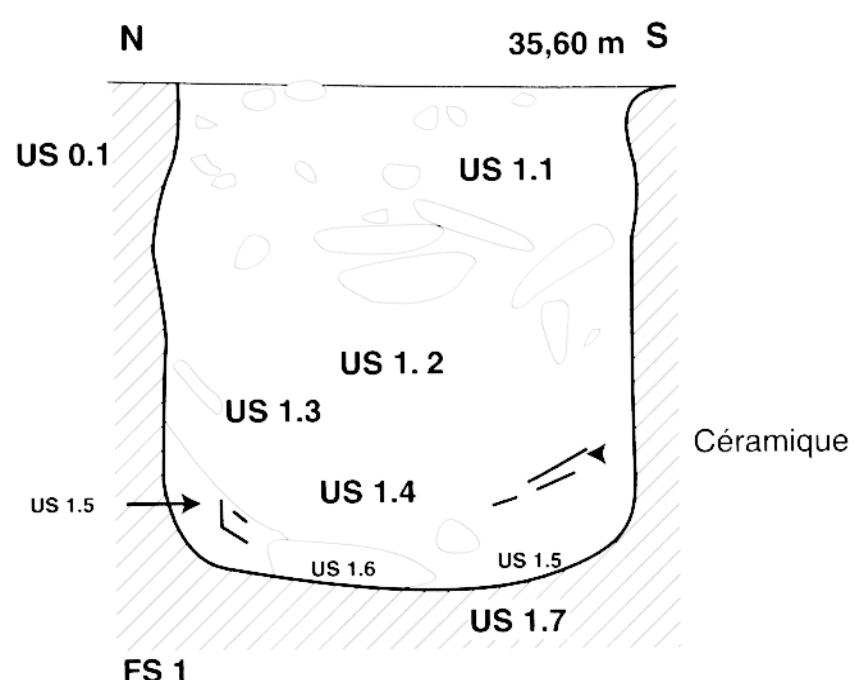

US 0.1 : substrat formé par les graviers de la terrasse fira.

US 1.1: limon argileux brun avec des plages de sables rouges amballant des graviers abondants et plus grossiers a la base.

US 1.2 : sédiment argilo-limoneux brun-rouge a structure grumeleuse renfermant des graviers et des charbons de bois.

US 1.3 : limon sableux de couleur brune avec graviers et petits galets. US 1.4: limon argileux mêlé à des poches dargile verte.

IS 1.5 : limon argileux brun fonce. très gravilloneux, à structure en agrígat.

US 1.6: pertubation animale, terrier.

IS 1.7 : creusement de la fosse. conservée - environ $5 \mathrm{~cm}$ - ne permettait aucune observation d'ordre stratigraphique ou morphologique. En planimétrie, l'anomalie apparaissait sous la forme d'une tache de forme ovalaire (fig. 3). Le remplissage se composait d’un limon brun-gris mêlé à des graviers. Elle correspond vraisemblablement à la base d'une fosse. Aucun mobilier n'a été retiré de ce lambeau de structure.

\section{La fosse FS.7}

Cette structure excavée présentait un plan circulaire d'un diamètre d'environ $0,95 \mathrm{~m}$. La section révèle laspect dissymétrique du creusement dont le fond est arrondi (fig. 3). Le comblement se compose d'un sédiment argilo-graveleux de teinte brun-gris. Compte tenu du faible volume du creusement, on remarque la forte densité des céramiques inclues dans le comblement. De toute évidence la fosse FS.7 ne peut pas s'apparenter à un silo arasé.

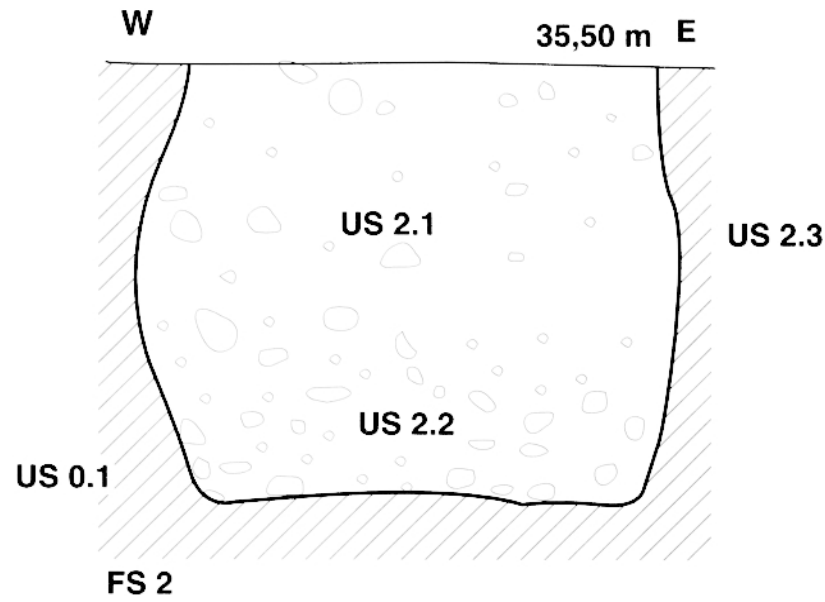

US 0.1 : substrat formé par les graviers de la terrasse Fya. US 2.1 : limon sableux brun avec graviers.

US 2.2 : limon sableux brun avec graviers denses.

is 2.3 : creusement de la fosse.

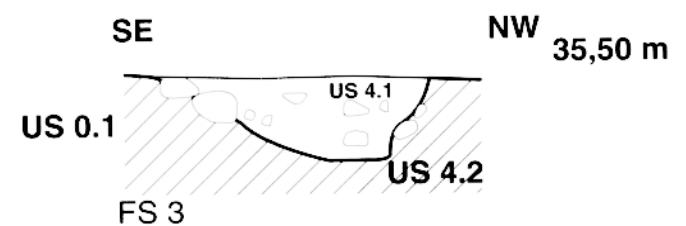

US 0.1: substrat forme par les graviers de la terrasse Fya.

US 4.1: limon sableux et graviers riches en charbons de bois et graines carbonisées mêlées à des plages de limon brun non rubéfièes.

LS 4.2 : creusement de la fosse (aucune trace de rubéfaction).

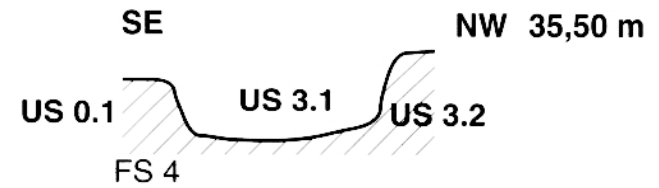

US 0.1 : substrat formé par les graviers de la terrasse Fya.

US 3.1: limon sableux et graviers riches en charbons de bois et graines carbonisées mêlées à des plages de limon brun non rubéfièes.

US 3.2: creusement de la fosse (aucune trace de rubéfaction). 


\section{La fosse FS.8}

Cette structure en creux présente un plan légèrement ovalaire dont le diamètre oscille entre 1,18 et $1,26 \mathrm{~m}$.
Apparue sous la forme d'une tache limono-graveleuse brune, son comblement n'était conservé que sur une puissance de $10 \mathrm{~cm}$ (fig. 3). Par sa morphologie, cette cuvette se rapproche de la structure FS.6.

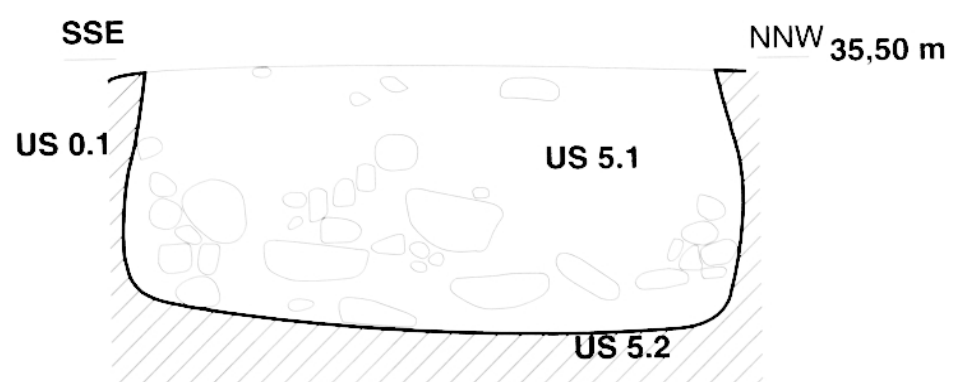

FS 5

FS 6

US 0.1

US 6.2

IS 0.1 : substrat formé par les graviers de la terrasse Fya IS 5.1: limon argileux brun gris avec graviers et blocs.

I'S 5.2 : creusement de la fosse.
Fosse tries arasice pas de coupe possible.

IS 0.1 : substrat forme par les graviers de la terrasse Iya.

IS 6.1 : limon argileux brun gris et graviers.

is 6.2 : creusement.
S

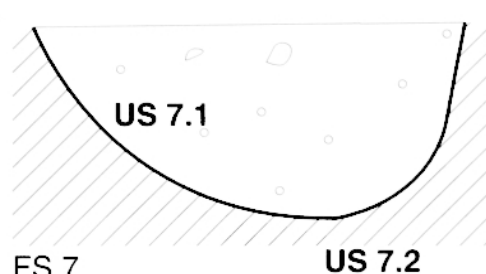

N

$35,45 \mathrm{~m}$

US 0.1
SE

\section{US 0.1} US 8.1

US 8.2
NO

$35,50 \mathrm{~m}$

IS 0.1 : substrat formé par les graviers de la terrasse lya IS 7.1 : limon argileux brun gris avec graviers.

IS 7.2 : creusement.

\section{W}

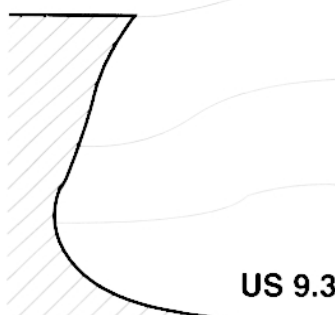

FS 9
US 9.1

US 9.2

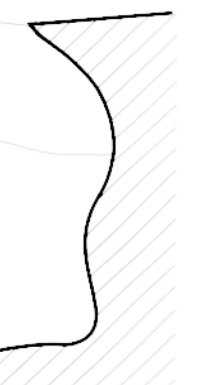

E $35,39 \mathrm{~m}$

US 0.1: substrat forme par les graviers de lat lerrasse liat IS 9.1: limon argileux gris avec graviers el blocs. IS 9.2 : limon argileux gris toncé avec graviers el blocs. IS 9.3: limon argileux noir avec graviers et blocs. IS 9.4: creusement.

Fig. 3 - Plan el coupes des structures FS.5. I:S.6. IS. 7 I:S.8 a lis.9. $\mathrm{FS} .=$ fosse. $\mathrm{LS}$. = unité stratigraphique (dessin L. I.oiselier). 


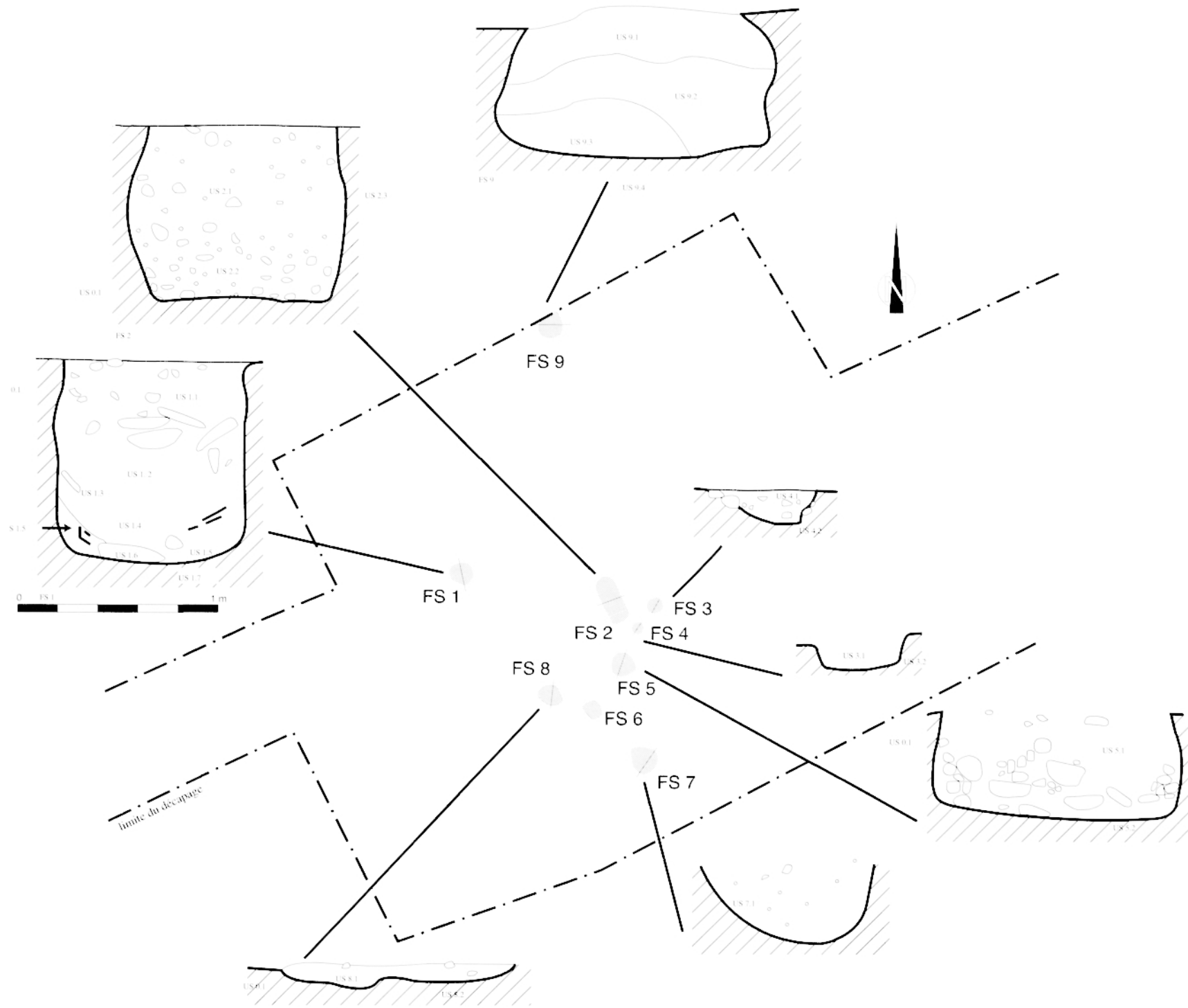

Fig. 4 - Plan général du site et position des structures en creux (dessin L. Caroma. L. Loiselier).

\section{La fosse FS.9}

La fosse FS.9, de plan circulaire, se présentait en limite du décapage sous la forme d'un indice dont le diamètre était compris entre 1,10 et $1,20 \mathrm{~m}$ (fig. 3). Le creusement était quant à lui conservé sur une épaisseur de $0.75 \mathrm{~m}$. Son profil montre que les parois décrivent un léger surplomb, accentué à l'est par une dépression. Le fond, bien que plat, présente quelques irrégularités. Par sa forme, cette structure s'apparente à un silo tronqué. Du point de vue du remplissage trois ensembles stratigraphiques ont été individualisés, bien que l'on constate une certaine homogénéité. À la base se trouvait une lentille (US.9.3) composée d'un sédiment limono-argileux noir, incluant des blocs et riche en graviers. La majeure partie du remplissage était constituée d'un sédiment limono-argileux de teinte gris foncé (US.9.2). Les caractères sédimentologiques du sommet de la structure ne présentent pas une modification sensible avec la précédente; seul le sédiment tend à s’éclaircir.

\section{Interprétation}

L'ensemble des indices archéologiques mis au jour correspond à des structures en creux. D'un point de vue taphonomique, on constate que le site a fait l'objet d'une dégradation importante liéc aux travaux agroculturaux. Il en résulte l'absence de conservation des sols ainsi qu'un arasement probable d'une portion des creusements. Les vestiges conservés ne peuvent alors correspondre qu'à des creusements de forte et moyenne amplitude. Il est probable que les trous ou calages de poteaux aient quant à eux été totalement détruits par les effets de l'érosion.

Parmi les 9 structures individualisées, trois d'entre elles peuvent être apparentées à des silos (fig. 4). Il s'agit des fosses FS.1, 5 et 9. En revanche, la structure 2 demeure 
plus difficile à interpréter. Son plan allongé permet d'écarter l'hypothèse d'un silo effondré. L'absence de conservation des structures superficielles ne permet pas de valider l'hypothèse d'une "cave" ou celle d'une structure en élévation de type grenier envisagée lors de la fouille (Viallet DFS). Les autres structures se singularisent par la faible amplitude de leur creusement. On remarquera la similitude du remplissage des structures 3 et 4 dont le comblement recèle des vidanges de foyers. Les autres aménagements sont extrêmement tronqués et ne possèdent aucun caractère discriminant. En raison de l'étroitesse de la surface décapée nous ne possédons qu'une vision très partielle du site du Bousquetas. Toutefois, on constate une concentration de structures en creux dans la partie médiane de la fouille. L'existence de silos indique la présence proche d'un habitat auquel certaines structures, telles que les cuvettes, peuvent appartenir. L'absence de vestiges relatifs a la présence de bâtiments en élévation doit être pondérée, notamment en raison des conditions de conservation du gisement. $\dot{A}$ ce propos, on peut s'interroger sur la présence de zones vierges de part et d'autre des structures fouillées.

La présence de structures de conservation, appuyée par l'étude des restes carpologiques, permet de mieux appréhender l'habitat dans son environnement. Il semble que parmi les raisons qui dictent l'implantation du site on puisse privilégier la situation géo-pédologique. En effet, le site se trouve à proximité du secteur alluvial de la basse terrasse de l'Hérault qui présente actuellement de bons sols, propices aux activités agricoles. Cette zone pouvait être soumise aux aléas des caprices du fleuve. Elle pouvait correspondre à des terroirs céréaliers.

\section{LE MOBILIER ET SA DATATION}

\section{L'échantillon}

Le site du Bousquetas a fourni 8 structures à partir desquelles nous avons dénombré 986 individus céraniques (décompte réalisé après remontage). Ce mobilier est de facto inégalement réparti. Le tableau ci-contre montre que les fosses 1 et 5 sont les mieux achalandées. Toutefois, la fragmentation qui caractérise ces deux structures limite l'approche typologique. D'autres structures ont livré un matériel que nous pouvons qualifier d'anecdotique (FS.3, 4, 8). D’une manière générale, la céramique des structures du Bousquetas se caractérise par un taux de fragmentation élevé, facteur limitant la portée des remontages ou restitutions des formes céramiques. Voir le tableau de décompte des mobiliers céramiques (tabl. 1). La fragmentation est définie en quatre classes.

\section{Inventaire des ensembles}

\section{La structure FS.1}

Cette structure a livré 294 tessons dont la plus grande partie est de petite dimension. Les tentatives de remontage n'ont pas permis de restituer la forme des

\begin{tabular}{|c|c|c|c|c|c|c|}
\hline structure & US. & esquilles & petits & moyens & grands & total \\
\hline FS.1 & $\begin{array}{c}1.2 \\
1.3 \\
1.1 \\
1.4 / 1.5 \\
\end{array}$ & $\begin{array}{r}26 \\
3 \\
14 \\
136 \\
\end{array}$ & $\begin{array}{r}9 \\
3 \\
2 \\
54\end{array}$ & $\begin{array}{r}4 \\
2 \\
1 \\
32 \\
\end{array}$ & 8 & $\begin{array}{r}39 \\
8 \\
17 \\
230\end{array}$ \\
\hline FS. 1 & total & 179 & 68 & 39 & 8 & 294 \\
\hline FS.2 & $\begin{array}{c}2 \\
\text { fond }\end{array}$ & $\begin{array}{r}20 \\
60 \\
2 \\
\end{array}$ & $\begin{array}{r}15 \\
35 \\
5\end{array}$ & $\begin{array}{l}4 \\
4 \\
1\end{array}$ & & $\begin{array}{r}39 \\
99 \\
8\end{array}$ \\
\hline FS.2 & total & 82 & 55 & 9 & & 146 \\
\hline IS. 3 & & & 1 & & & 1 \\
\hline FS. 4 & & & & 1 & 1 & 2 \\
\hline FS. 5 & & 120 & 125 & 45 & 12 & 302 \\
\hline FS.7 & 1 & $\begin{array}{r}13 \\
8\end{array}$ & $\begin{array}{r}20 \\
7\end{array}$ & 2 & & $\begin{array}{l}33 \\
17\end{array}$ \\
\hline FS.7 & total & 21 & 27 & 2 & & 50 \\
\hline FS.8 & & 2 & & & & 1 \\
\hline FS.9 & $\begin{array}{l}1 \\
2\end{array}$ & $\begin{array}{r}23 \\
43 \\
3\end{array}$ & $\begin{array}{r}38 \\
37 \\
4\end{array}$ & $\begin{array}{r}13 \\
15 \\
1\end{array}$ & $\begin{array}{l}7 \\
5 \\
1\end{array}$ & $\begin{array}{r}81 \\
100 \\
9\end{array}$ \\
\hline FS.9 & total & 69 & 79 & 29 & 13 & 190 \\
\hline TOTAL & & 473 & 355 & 125 & 34 & 986 \\
\hline
\end{tabular}

Tabl. 1 - Décompte des mobiliers céramiques. La fragmentation est detinie en quatre clatsies.

récipients. Les bords sont très nombreux (fig. 5). II s'agit probablement, pour les plus petits, de fragments d’écuelles au profil fermé. D’autres s’apparentent à des jarres ou des récipients de grand volume. Cette catégorie est représentéc par un vase au profil fermé portant une languette. La datation réalisée pour la base du remplissage - US.1.4/1.5 - fournit les résultats suivants Ly-468(Oxa) $4255 \pm 50$ B.P., soit 2990-2661 cal. bc, avec des probabilités maximum de -2884-2789-2709.

\section{La structure FS.2}

Le mobilier de cette structure est essentiellement représenté par des éléments de bords (fig. 5, n" 17 à 27). Leur taux de fragmentation est trop important pour que l'on puisse déterminer leurs formes. On remarque la présence d'un tesson possédant deux languettes superposées, pouvant signifier une attribution au Néolithique final de type Vérazien (fig. 5, n"28).

\section{La structure FS.3}

Présence d'un tesson portant un cordon lisse sous le bord (fig. 5, n" 30).

\section{La structure FS.4}

Cette structure n'a livré qu'un seul récipient (fig. 6 . $n^{\prime \prime} 1$ ). Il s’agit d'une petite écuelle au profil fermé et arrondi. Ce type de récipient du fonds commun se rencontre dans des sites tels que celui de Saint-Paul à Mèze, Hérault (Rouquette, Montjardin, 1991) ou celui 

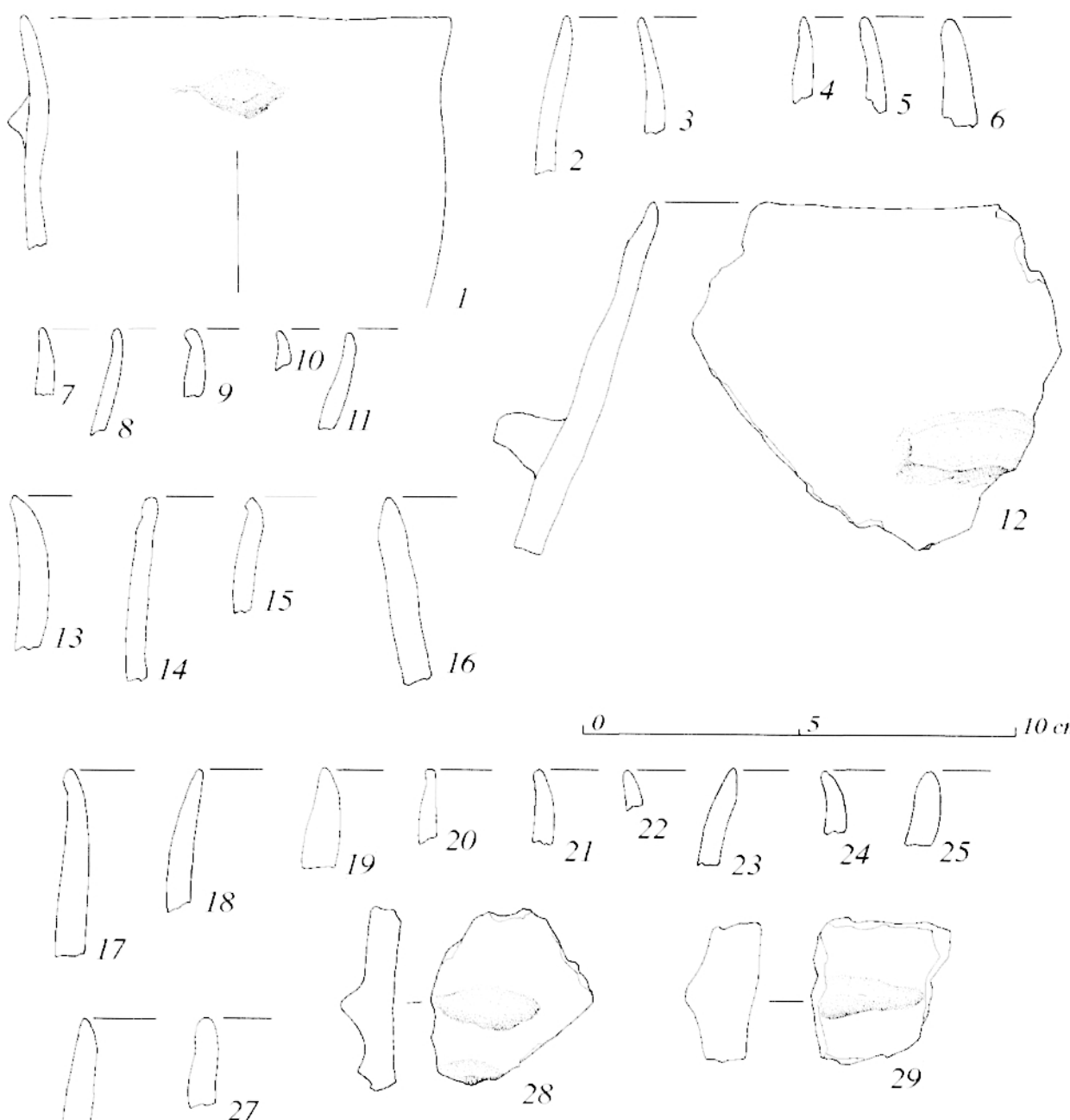

$0+15$ $10 \mathrm{~cm}$
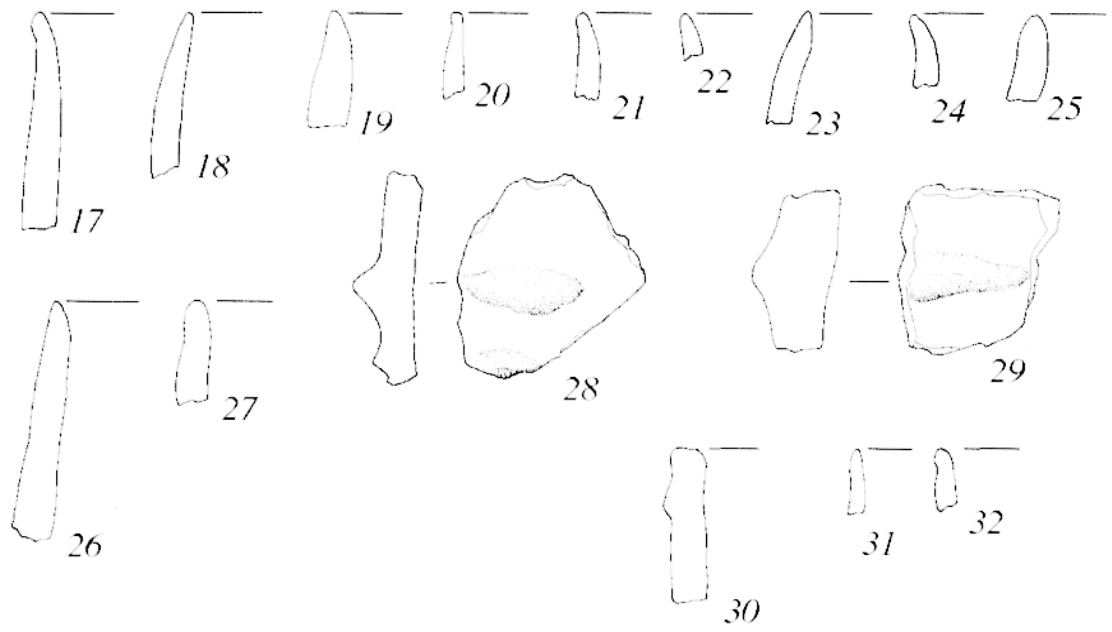

0 5 $10 \mathrm{~cm}$

Fig. 5 - Mobilier céramique des structures lFS.1 (1 a 16). FS.2 (17 à 29). FS.3 (30), FS.7 (31, 32). dessin L. Carozza.

de la Croix-Vieille à Montblanc, Hérault (Espérou, Roques, 1994).

\section{La structure FS.5}

Le mobilier de cette structure est suffisamment caractéristique. Les écuelles ornées de pastillage au repoussé (fig. $6, n^{\circ} 4,6$ et 7 ) fournissent des éléments de comparaison dans un environnement proche. Ce type de décor est connu durant le Néolithique final dans la région de Cabrières comme à la grotte du Broum (Ambert, Barges, 1991) ou sur le site de Roquemengarde (information Guilaine, Coularou). La céramique du fonds commun est représentée par une petite marmite au profil vertical et munie d'un mamelon (fig. 6, n" 2). On remarque également la présence d'une anse. D’une manière générale, le mobilier de cette structure peut être attribué au Néolithique final/Chalcolithique, tel qu'il se singularise dans la vallée de l'Hérault. En ce sens, il présente des similitudes avec le mobilier issu du site des Hermes à Bélarga, bien que les éléments de type fontbouïsse en soient absents.

\section{La structure FS.7}

Cette structure n’a livré que deux éléments de bords (fig. $5, n^{\prime \prime} 31,32$ ).

\section{La structure FS.9}

Le mobilier de cette structure, bien qu'indigent (fig. 7), nous fournit des renseignements. Outre quelques éléments de préhension, on remarque la présence de deux tessons ornés de pastillages au repoussé. Comme 
nous l'avons précisé précédemment, ce caractère peut être rapporté au Néolithique final/Chalcolithique de la région qui nous intéresse.

\section{Attribution chronologique}

La présentation de chacun des ensembles mobiliers a permis de se rendre compte de l'extrême indigence des données. Pris globalement, le mobilier du site du Bousquetas semble toutefois homogène et devoir être attribué au Néolithique final. Cette homogénéité n’exclut pas, bien entendu, des nuances chronologiques internes. La présence des décors pastillés au repoussé constitue un élément de datation. Sans développer les multiples corrélations observées, ce thème décoratif de la céramique, qui apparaît dès le
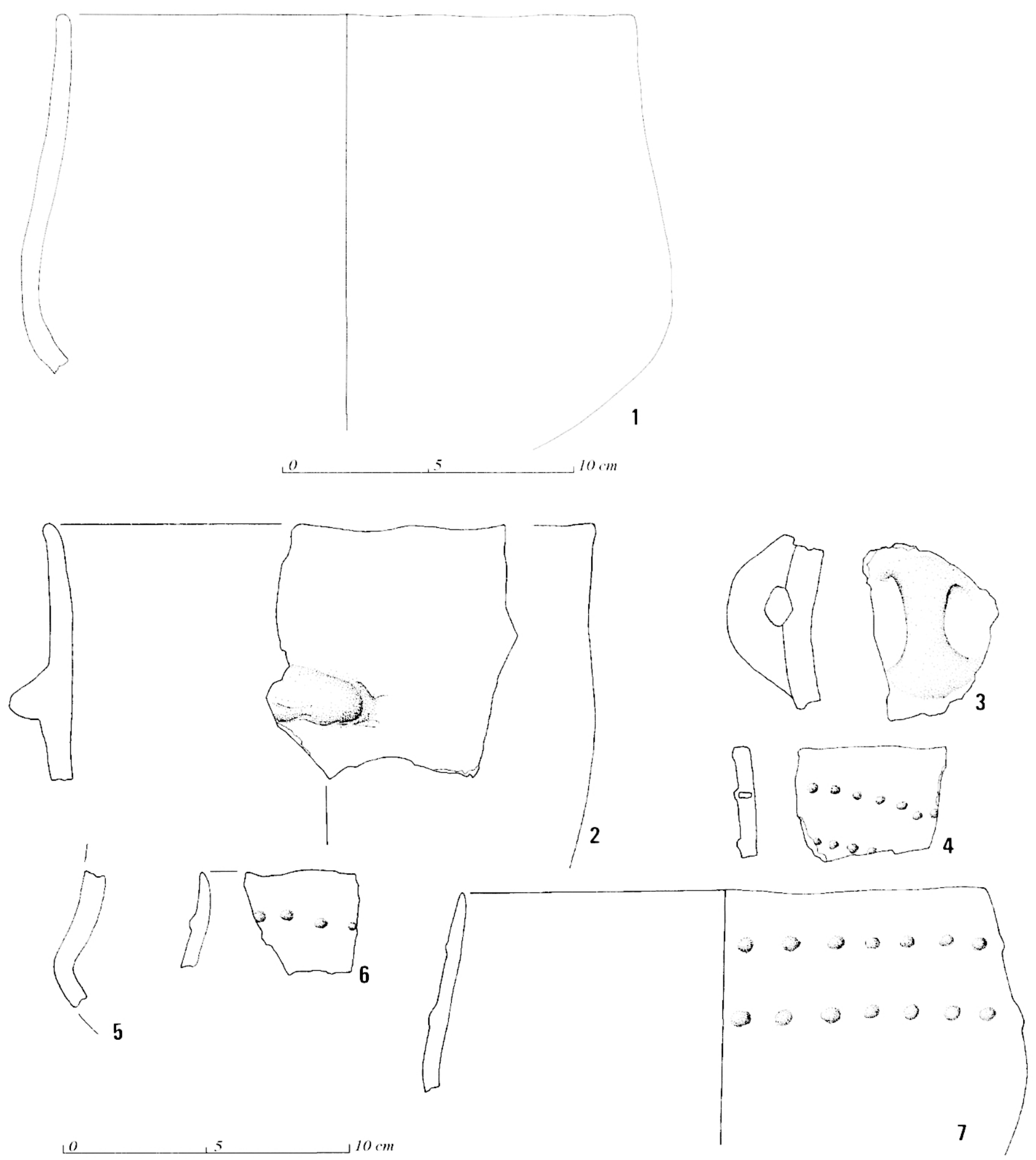

Fig. 6 - Mobilier céramique des structures FS.4 (n"1) et FS.5 (2 à 7) dessin L. Carozza. 
48

Laurent CAROZZA. Pascal VIALFT ave la collaboration de Anne BOUCHETTE et Pascale CHEVILLOT
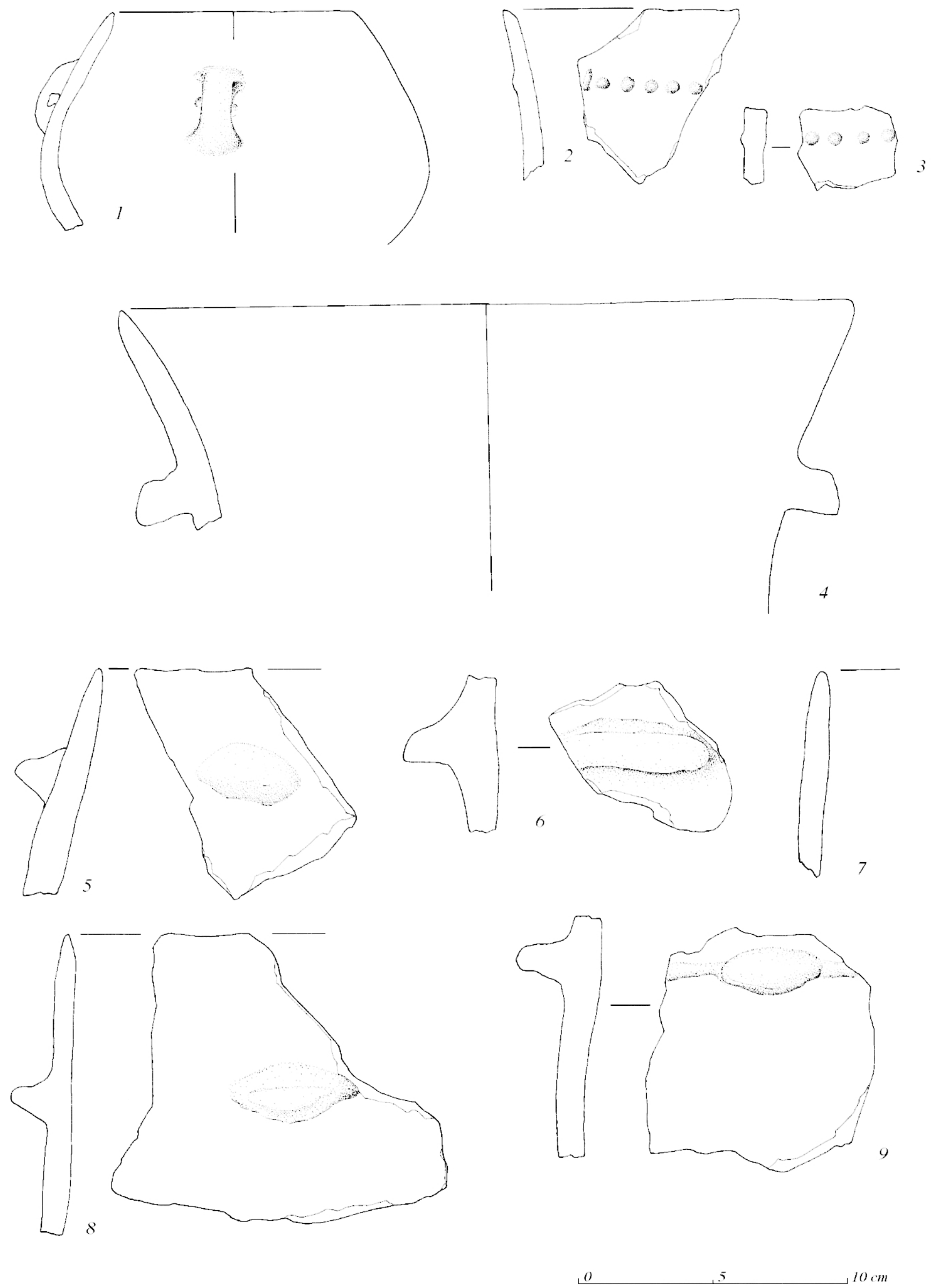

Fig. 7 - Mobilier de la structure FS. 9 dessin L. Carozza.

Bulletin de la Sociète Préhistorique Française

1999, tome 96, n"1, p. $39-51$ 
Néolithique final, constitue un trait caractéristique de la région de la moyenne vallée de l'Hérault comme à Roquemengarde (Guilaine et al., 1988) ou sur le site de la Croix-Vieille (Espérou et al., 1996). Le polymorphisme de la culture matérielle rend ici les notions de chronologie délicates à manier, surtout lorsque l'échantillon de référence s'avère aussi pauvre que celui du Bousquetas. On constatera simplement l'absence de décors fins, seuls à même d'être corrélés avec des faciès céramiques des groupes limitrophes. Si l'on prend en compte la datation absolue obtenue pour la structure FS.1, on constate que les ensembles de la CroixVieille - 3021-2619 bc - (Montblanc, Hérault) ou de la Croix-de-Fer - 2900-2590 bc - (Espondeilan, Hérault) se trouvent dans une fourchette comparable au site du Bousquetas, cest-à-dire dans le premier quart du $3^{\circ}$ millénaire avant notre ère. Le Bousquetas peut également être rapproché de la fin de l'occupation du site de Roquemengarde - 2860-2460 bc - (Saint-Pons-deMauchiens, Hérault). D'une manière plus large, il existe une forte probabilité pour situer l'occupation du site du Bousquetas dans un intervalle de 2900-2700 bc.. et donc contemporaine de la phase moyenne ou classique du Vérazien et de la fin du Ferrières.

\section{ÉTUDE ARCHÉOBOTANIQUE DES STRUCTURES} FS.3, FS.4 et FS.2 (Anne Bouchette)

Les fosses FS.3 et FS.4 ont fait l'objet de prélèvements en vue d’une analyse archéobotanique. Ce choix a été motivé par la nature même du remplissage essentiellement composé ici de vidanges de foyers. Une troisième structure, la fosse FS.2, a également livré des paléosemences carbonisées. L'étude anthracologique, réalisée par L. Chabal, a en effet permis d'isoler, pour un total de 70 litres de terre tamisée, deux grains de Céréales: un d'Orge (Hordeum vulgare) et un de blé tendre/dur (Triticum aestivum/durum) (fig. 8).

Les prélèvements effectués dans les niveaux cendreux des fosses FS.3 et 4 sont très pauvres en charbons de bois, mais nettement plus riches en paléosemences: 2508 fruits et graines ont été recueillis pour une quantité de terre tamisée respectivement de 30 litres pour la fosse 3 et de 20 litres pour la fosse 4 . Ces chiffres ne rendent, cependant, pas compte de la concentration des échantillons en paléosemences. En effet, seuls 10 litres de sédiments, pour chacune des deux fosses, ont lait l'objet d'une étude exhaustive (fig. 1, Lab. N" 4 et 5). Une première étude partielle avait livré environ 500 paléosemences. L'étude approfondie n’a pas permis de mettre en évidence de nouveaux taxons. Mais, il a été possible de mieux quantifier la densité en restes végétaux de ces niveaux de remplissage. Celle-ci est élevéc puisquelle atteint environ 200 restes par litre.

Les deux fosses présentent une répartition et un spectre floristique semblables (fig. 9), caractérisés par une très nette prédominance de l'orge, de l'ordre de $90 \%$. La forte densité en restes végétaux des échantillons permet de penser que l'on est en présence de céréales qui auraient été carbonisées puis jetées dans des fosses.

La quasi-absence de charbons de bois renforce cette hypothèse. L'association d'un remplissage cendreux et d'un pourcentage élevé de restes végétaux a, par ailleurs, souvent été observée (par ex., Stika, 1996). Deux autres céréales sont présentes avec des pourcenlages légèrement différents dans les deux fosses : le blé tendre/dur (Triticum aestivum/durum) et l'engrain (Triticum monococ'um). Le premier est mieux représenté dans la fosse 3 que le second. Á l'inverse, l'engrain est légèrement plus abondant dans la fosse 4 que le blé tendre/dur et il y est représenté à double titre: par des caryopses et des bases d'épillets.

La présence d’orge et de blé dans chacune des deux fosses indique la culture de ces céréales dans l'environnement du site. Le mélange de ces différentes céréales, en des proportions très différentes, pourrait être un indice de rotation de cultures. L’orge aurait été cultivée dans des champs précédemment ensemencés en blé. Mais, il pourrait également résulter du stockage. Dans ce cas, l'orge pourrait avoir été stockée dans un endroit qui aurait précédemment contenu du blé.

\begin{tabular}{|c|c|c|c|c|c|c|c|c|}
\hline & Organe & $\begin{array}{c}\text { Lab. } \\
\text { n } 1+4\end{array}$ & $\begin{array}{l}\text { Lab. } \\
n+4\end{array}$ & $\begin{array}{c}\text { Lab. } \\
\text { n } 2+5\end{array}$ & $\begin{array}{l}\text { Lab. } \\
\text { n } 5\end{array}$ & $\begin{array}{l}\text { Lab. } \\
n 3\end{array}$ & Somme & $\%$ \\
\hline Datation & & \multicolumn{2}{|c|}{ Néo Final/Chalco } & \multicolumn{2}{|c|}{ Néo Final/Chalco } & Néo Final/Chalco & & \\
\hline Structure & & \multicolumn{2}{|c|}{ Fosse cendrier } & \multicolumn{2}{|c|}{ Fosse cendrier } & Fosse/cave & & \\
\hline $\mathrm{FS}$ & & \multicolumn{2}{|c|}{3} & \multicolumn{2}{|c|}{4} & & & \\
\hline US & & \multicolumn{2}{|c|}{3.1} & \multicolumn{2}{|c|}{4.1} & 2 & & \\
\hline Volume tamise (en litres) & & 30 (total) & dont 10 & 20 (total) & dont 10 & 70 & & \\
\hline \multicolumn{9}{|c|}{ TAXONS } \\
\hline Hordeum vulgare (orge) & Caryopse & 1093 & 874 & 1151 & 976 & 1 & 2245 & 89,44 \\
\hline Triticum aestivum / durum (blé tendre/dur) & Caryopse & 137 & 80 & 13 & 9 & 1 & 151 & 6,01 \\
\hline Triticum monococcum (Engrain) & Caryopse & 7 & 3 & 74 & 61 & 0 & 81 & 3,23 \\
\hline Triticum monococcum (Engrain) & Base d’épillet & 0 & 0 & 7 & 5 & 0 & 7 & 0,28 \\
\hline Triticum spec. (blé) & Caryopse & 6 & 0 & 10 & 4 & 0 & 16 & 0,64 \\
\hline Cf. Hordeum vulgare (cf. orge) & Caryopse & 8 & 0 & 0 & 0 & 0 & 8 & 0,32 \\
\hline Cerealia Céréales (en grammes) & Caryopse & 7,27 & 7,27 & 7,14 & 7,04 & 0 & 14,41 & - \\
\hline Fabaceae (légumineuses) & Graine & 2 & 0 & 0 & 0 & 0 & 2 & 0,08 \\
\hline SOMME & & 1253 & 957 & 1255 & 1055 & 2 & 2510 & 100 \\
\hline
\end{tabular}

Fig. 8 - Liste des taxons déterminés. 


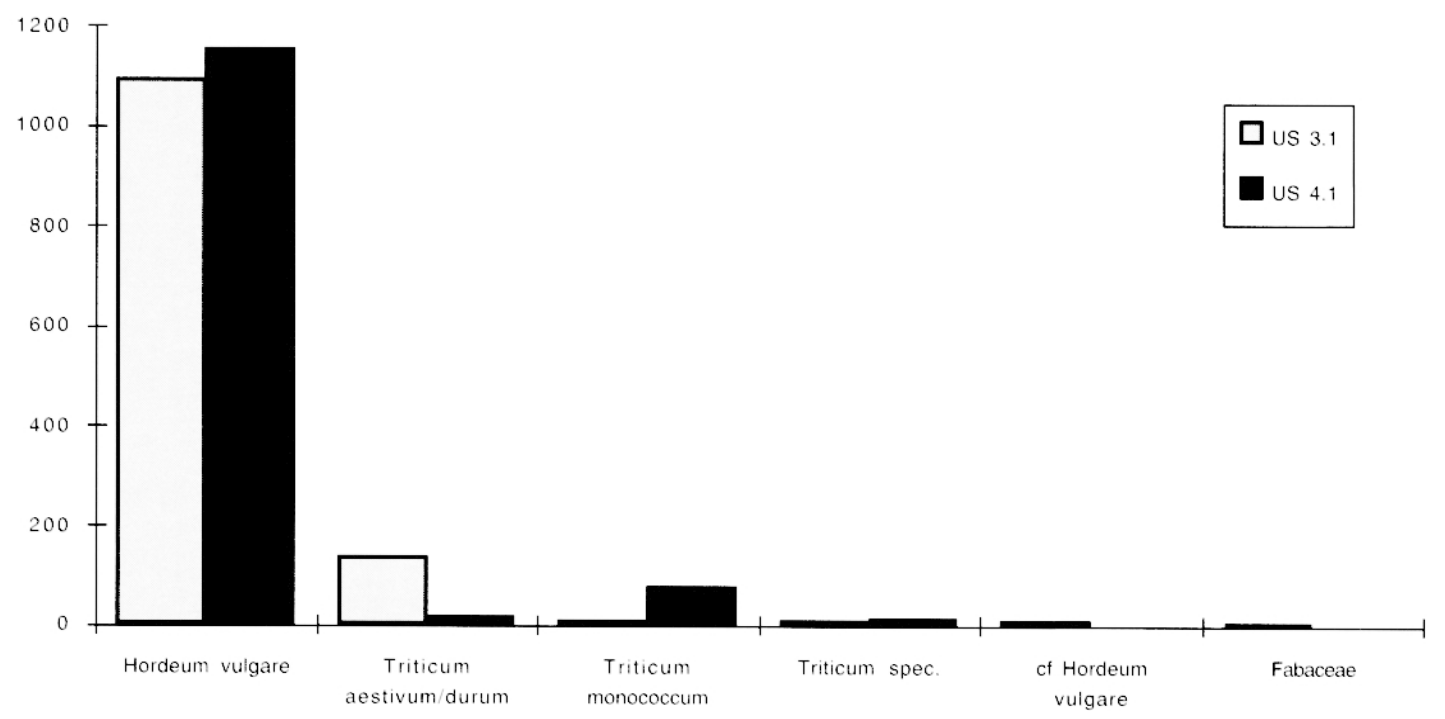

Fig. 9 - Distribution des taxons dans les structures FS.3 et 1:S.4.

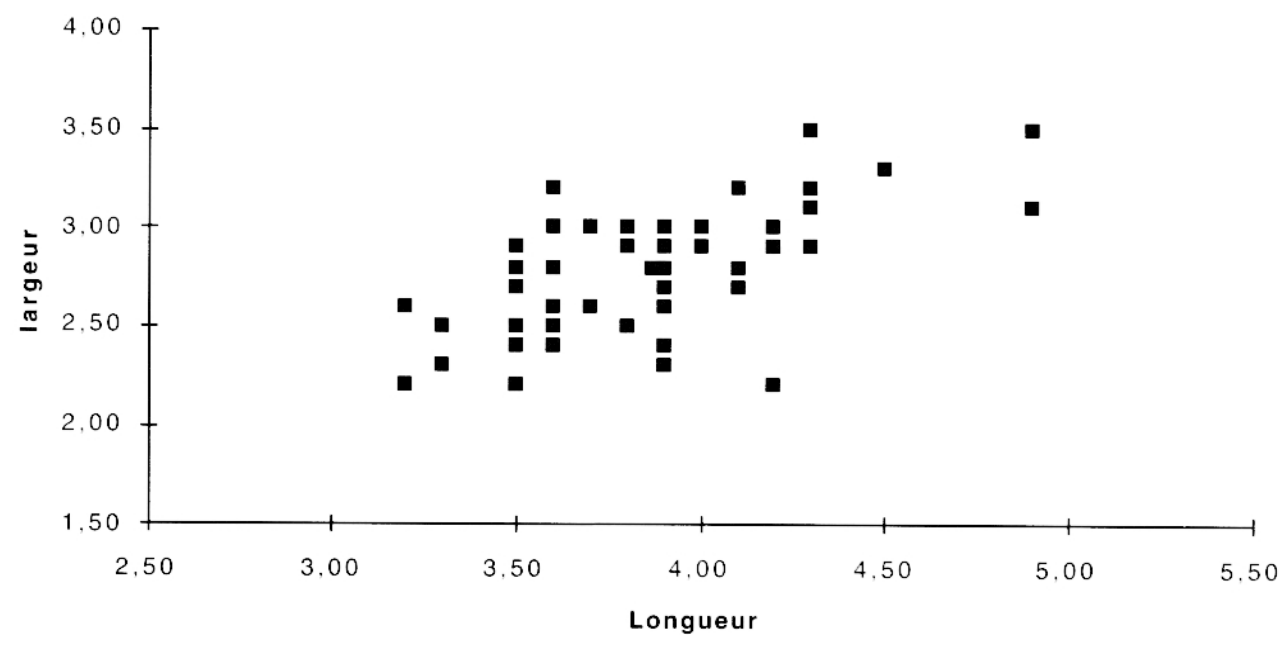

Fig. 10 - Indice biométrique des grains d’orge carbonisès $(\mathrm{N}=50)$.

L'état de conservation des grains est dans l'ensemble médiocre, voire mauvais. Ce caractère est plus marqué pour les caryopses de la fosse 4 , particulièrement érodés. La fragmentation des grains est également importante, ceci dans les deux fosses. C'est pourquoi, les fragments de Cerealia n'ont pas été comptabilisés. Les mesures effectuées sur les grains d'orge soulignent leur petite taille (fig. 10). D'aspect fusiforme, ils possèdent un sillon ventral très superficiel et, en coupe transversale, un aspect subcirculaire. De manière générale, ils ont une forme trapue.

Ces trois espèces sont bien attestées dans le sud de la France au Néolithique final/Chalcolithique. Elles le sont déjà pour le Néolithique moyen et, pour l'orge et le blé tendre/dur, au Néolithique ancien (Marinval, Ruas, 1991). Le blé tendre/dur est une espèce à grains nus, panifiable, réputée plus exigeante du point de vue sol que l'engrain. L'absence de toute détermination spécifique de messicoles ne permet toutefois aucune approche de la mise en valeur des terroirs. Situé à proximité, le site d'Aspiran le Bernat, daté de la fin du
Néolithique moyen, n’a livré que très peu de paléosemences. Les deux espèces attestées sont néanmoins l'orge et le blé tendre/dur.

\section{REMERCIEMENTS}

Cette opération à pu être réalisée avec le concours de GDF. Les auteurs tiennent à remercier tout particulièrement Monsieur Jérôme Kotarba, coordinateur pour l'AFAN de l'opération Gazoduc " artère du Midi " pour son soutien, ainsi que Monsieur Alain Chartrain, Conservateur au Service Régional de l'Archéologie de Languedoc Roussillon.

\section{NOTE}

(1) Tests effectués par Christian Pradiès et Christian Gaudillère, équipe de tamisage mise en place pour toutes les opérations de la vallée de l'llérault. 


\section{RÉFÉRENCES BIBLIOGRAPHIQLES}

IESPFROU J.-L. (1993) - La structure métallurgique chalcolithique de Roque-Fenestre (Cabrieres. Hérault), Archoologie' e'n Lansueder, n" 17. p. 32-46.

I:SPEROU J.-L... ROQUI:S P. (1994) - L enceinte chalcolithique de la Croix Vieille a Montblanc (Hérault). Prémiers résultats. Bulletin de la Socicte Prihistorique Française, 1. 91, n' 6, p. $422-428$.

IESPI:ROU J.-L., ROQUISS P., PFRNAU J. (1996) - Loncinte chalcolithique de la (roix Vicille a Montblanc (Hérault). La structure n" 8. Bulle'tin de la Soctiote Prehistorique Pransaise. t. 93. n"4. p. $5+9-5(0)$.

(i ASCO J. (1992) - l a chronologie absolue du Néolithique final el du Chalcolithique en Languedoc Méditerrancen, in: l.c Chalcolithique an languedec. se's relations extra-rigionales. Archeologie en Langlledoc, Ilommage au Dr Jean Arnall, p. 217-225.

(iASCO J.. (jUTHl:R/ X. (1986) - Origine el structure du Nélithique final en Languedoc mediterranesn: la céramique. in:

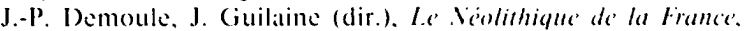
Picard, p. 379-390.

(iUILAINE: J., COULAROU J.. BRIOIS F., RIVENQ C. (1988)L habitat neolithique de Roquemengarde (Saint-Pons-deMatuchicns. Héraula) Premiers éléments sur le dispositif d'enceinte, in: A. d'Anna, X. Gutherz (dir.). Enceinte's, habirats cointures et habitats perche's du Neolithique' au Bronae ancie'n, p. 21-29.

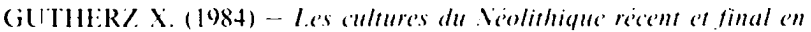
langucdec oriental. These de doctorat. Université de Provence. 2 tomes, 345 p., 247 tig. al 9 pl.

JANIN T. (1993) - Les sociétés Néolithiques dans le bassin de lHerault, Bulletin du GREC, n" 67-68-69. p. 5-21.

M.IRINVAI. P.. RUIAS M.-P. (1991) - Alimentation végétale el agriculture d'apres les semences archéologiques (de 9000 av. J.-C. au
XV" siecle), in: J. Guilaine (dir.), Pour une archoologie agraire. id. A. Colin, p. $409-439$.

ROLIQLETTE D.. MONTJARIDIN R. (1992) - Le Chalcolithique dans le secteur de Thatu-(jardiole, in: le Chalcolithique e'n langurdoce se's relations extra-regionales, Archéologie en Languedoc. Ilommage au Dr Jaan Arnal. p. 259-276.

STIKA H.-P. (1996) - Vorgeschichliche Pflanzenreste aus HeilhromnKlingenters. Materialhefte zur Archäologic, ed. Konrad Theiss. $230 \mathrm{p}$.

VAQI:IR J., CORNI:JO A.. C ARRFRF I. A BOLIRIIIS J.-R. (1993)le gisement Veratien de Montrose a Tourbes (llerault). tricher-

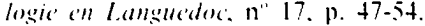

Laurent CAROZZA

U.M.R. 150 C.N.R.S.-E.H.E.S.S.-U.P.S. 39, allées Jules-Guesde, 31000 Toulouse

Pascal VIALET A.F.A.N. MEDITERRANÉE 12, rue Régale, 30000 Nîmes

Anne BOUCHETTE A.F.A.N. GRAND SUD-OUEST 12. rue Marcelin-Berthelot, 33270 Floirac

Pascale CHEVILLOT A.F.A.N. MEDITERR ANÉE 12. rue Régale, 30000 Nîmes 